PALABRAS CLAVE

Delito

Medición

Análisis de datos

Delincuentes

Distribución geográfica

Análisis demográfico

Estadísticas demográficas

Modelos matemáticos

Chile
REVISTA CEPAL $98 \cdot$ AGOSTO 2009

\section{Caracterización socioeconómica y espacial de la criminalidad en Chile}

\author{
Mauro Gutiérrez, Javier Núnez y Jorge Rivera
}<smiles>[CaH]</smiles>

n supuesto de gran parte de los estudios empíricos sobre

la criminalidad es que víctimas y victimarios conviven en una misma unidad geográfica, lo cual implica utilizar los rasgos socioeconómicos del lugar de residencia del denunciante como factores determinantes del crimen. En este estudio se ofrece una mirada alternativa que consiste en medir la criminalidad sobre la base de la proporción de imputados en la población total, considerando las características de las comunas de procedencia pertinentes como causas socioeconómicas

Mauro Gutiérrez

Tesista de Doctorado,

Departamento de Economía,

Universidad de Chile

œ gutierrez_mauro@hotmail.com

Javier Núñez

Profesor Asociado,

Departamento de Economía,

Universidad de Chile

œjnunez@econ.uchile.cl

Jorge Rivera

Profesor Asociado,

Departamento de Economía,

Universidad de Chile

๑jrivera@econ.uchile.cl de la delincuencia. Se concluye que los imputados presentan un grado importante de movilidad espacial. En el caso de los delitos de motivación económica, las evidencias respaldan parcialmente los postulados de Becker. Por último, se muestra que el número de imputados tiende a ser más significativo en las comunas de menor ingreso, con mayor presencia policial, en que predominan las zonas urbanas de mayor escolaridad y del norte del país, lo que en cierta medida concuerda con los resultados de otros estudios sobre Chile. 


\section{I}

\section{Introducción}

En la última década, Chile ha presentado tasas crecientes de criminalidad, fenómeno que se ha convertido en una de las principales preocupaciones de la población. Los delitos de robo y hurto, por ejemplo, aumentaron un $12 \%$ y un $13 \%$ anual, respectivamente, mientras que los homicidios y los delitos relacionados con las drogas lo hicieron un $2 \%$ y un $33 \%{ }^{1}$ Naturalmente, esta tendencia ascendente de la delincuencia ha despertado inquietud en la ciudadanía, que la considera uno de los problemas más importantes que enfrenta la sociedad chilena en la actualidad. ${ }^{2}$ No obstante lo anterior, las investigaciones y el conocimiento de los factores determinantes de la criminalidad en el país son aún escasos y limitados.

Desde la contribución inicial de Ehrlich (1973), los estudios empíricos y econométricos sobre la criminalidad han permitido avanzar considerablemente en la comprensión de algunas de sus causas fundamentales. ${ }^{3}$ En la mayoría de estas publicaciones, la criminalidad se mide según el número de denuncias y se utilizan las características socioeconómicas de las zonas geográficas pertinentes como variables determinantes de ella, bajo el supuesto tácito de que los victimarios provienen de la misma localidad que las víctimas. Aunque esta aproximación parece razonable cuando se trata de áreas geográficas extensas, su poder explicativo se reduce cuando estas son heterogéneas o si el lugar en que se denunció el delito no coincide con la localidad de origen del victimario. ${ }^{4}$

$\square$ Los autores agradecen los comentarios y sugerencias de un evaluador anónimo de Revista CEPAL sobre una versión anterior del presente artículo, así como del profesor José Miguel Benavente y de los participantes del Encuentro de la Sociedad de Economía de Chile (SECHI), realizado en septiembre de 2008. Este trabajo fue financiado parcialmente por el Fondo Nacional de Desarrollo Científico y Tecnológico (FONDECYT), de conformidad con el Proyecto No 1070856 de 2007, "Un análisis del mecanismo de licitación de servicios de defensa: incentivos perversos, oferta criminal". Se agradece, además, el apoyo del Instituto Milenio Sistemas Complejos de Ingeniería.

${ }^{1}$ Véase Anuario de estadísticas criminales 2008 de la Fundación Paz Ciudadana. La tasa de crecimiento anual corresponde al número de denuncias realizadas.

2 De acuerdo con la Encuesta Nacional de Seguridad Ciudadana 2005, el $29 \%$ de la población señaló que la delincuencia y el tráfico de drogas son los principales problemas de la actualidad nacional. Por otra parte, según Londoño, Gaviria y Guerrero (2000), el costo de los delitos de violencia en América Latina varía entre un 5\% y un $13 \%$ del PIB.

${ }^{3}$ Otros aportes econométricos tempranos e influyentes fueron los de Wolpin (1980) y Dryden Witte (1980).

${ }^{4}$ Este tipo de enfoque podría implicar que existe una relación proporcional entre el nivel de ingresos y el de criminalidad. Para
En este trabajo se procura abordar las limitaciones mencionadas, al considerar la procedencia geográfica de los imputados en vez del lugar de inscripción de la denuncia por parte de las víctimas. A nuestro entender, se trata del primer esfuerzo de esta naturaleza en Chile y América Latina. La motivación conceptual de este enfoque es que si la propensión a efectuar delitos depende del entorno físico, social y económico de los individuos, un análisis basado en la localidad de origen de los victimarios y de las características pertinentes podría ofrecer nuevas luces sobre los factores determinantes de la criminalidad en el país.

Para esos efectos, se utilizó la llamada base de datos de imputados de la Defensoría Penal Pública (DPP) de Chile correspondiente a los años 2005 y 2006. La unidad geográfica de análisis fue la comuna y se consideró el número de imputados por cada 100.000 habitantes de ellas, según el tipo de delitos registrados. ${ }^{5}$

Aunque los imputados no son necesariamente victimarios de delitos, el análisis se basó en la idea de que son una medida imperfecta pero cercana, dado que una proporción importante y hasta cierto punto constante de ellos son declarados culpables de los delitos por los cuales fueron procesados.

Uno de los resultados del estudio es que, en el caso de la mayoría de los delitos, existen comunas en que no hay imputados que procedan de ellas. Este hecho

una discusión más detallada al respecto, véase Rivera, Núñez y Villavicencio (2004)

${ }^{5}$ La base de datos de imputados de la DPP contiene antecedentes de prácticamente todas las causas penales que se han desarrollado en los últimos años en el país. La información sobre cada imputado incluye, entre otros, edad, género, ingreso declarado, delito por el cual fue acusado, duración del proceso y sanción decretada por el juez. En particular, se registran el domicilio y la comuna de origen del imputado, así como el lugar donde habría cometido el supuesto delito. Por otra parte, desde el punto de vista administrativo, Chile tiene 15 regiones, 51 provincias y 342 comunas. La población comunal media se acerca a los 50.000 habitantes, con una alta dispersión geográfica. Todos los datos socioeconómicos sobre las comunas que se utilizan en este trabajo corresponden a la Encuesta de Caracterización Socioeconómica Nacional (CASEN) que se aplica cada dos años en todo el país, desde fines de la década de 1980. Mediante estas encuestas se recopilan algunos antecedentes significativos sobre la población a nivel comunal, tales como estructura etaria, nivel de ingresos, características y composición de los hogares. En este trabajo se utilizaron los resultados de la CASEN 2006. Para mayores detalles, véase [en línea] www.mideplan.cl. 
implicó la necesidad de desarrollar un procedimiento que permitiera abordar separadamente el problema del número de imputados que pertenecen a cada comuna y el de imputados registrados efectivamente en ella, para lo cual se empleó un modelo Heckit calculado según máxima verosimilitud. Se realizaron estimaciones para diversos tipos de delitos, utilizando como variables explicativas los ingresos legales o ilegales, las probabilidades de sanción y las características de la comuna de origen de los imputados. Este es el llamado modelo de selección que se describe más adelante.

En forma complementaria, en este trabajo se desarrolló y estimó el denominado modelo de participación de la criminalidad, a fin de establecer los factores determinantes de la imputación en función de las variables que se acostumbra emplear en los estudios sobre el tema y de la comuna de origen de los imputados. Esto último es, precisamente, uno de los aportes novedosos del presente trabajo y que lo distinguen de los esfuerzos ya realizados. Las conclusiones así obtenidas coinciden con los resultados de otras investigaciones efectuadas en Chile a partir de datos relacionados con las denuncias a nivel regional (Rivera, Núñez y Villavicencio, 2004).

El trabajo está estructurado de la siguiente manera. Después de esta introducción, en la sección II se presentan algunos hechos estilizados sobre el comportamiento de las denuncias según tipo de delitos que surgen al considerar como unidad de análisis la procedencia geográfica de los imputados. En la sección III se describe el modelo teórico y econométrico aplicado en este trabajo, además de los datos utilizados en las estimaciones. Por último, en las secciones IV y V se presentan los resultados y las conclusiones del estudio, respectivamente. En el anexo se adjuntan algunos cuadros que contienen los resultados econométricos discutidos en el cuerpo del texto, así como una discusión más detallada de la relación entre criminalidad e imputabilidad que sustenta nuestra opción por esta última para evaluar la delincuencia a nivel comunal.

\section{II}

\section{Hechos estilizados}

En esta sección se ofrecen y discuten varios hechos estilizados sobre el lugar de procedencia de los imputados en Chile a nivel comunal, adelantando algunos temas que se abordarán en los apartados siguientes. El cuadro 1 muestra el número de comunas en que hubo residentes imputados por los delitos señalados y el porcentaje que representan respecto de las 335 de que se dispone de información sobre el año 2006. Los datos revelan que hay delitos en que la tasa de "no participación comunal" alcanza el $31 \%$ (homicidio) y otros más difundidos geográficamente (hurto y lesiones).
Esta característica resulta novedosa, ya que si bien en estudios anteriores se concluyó que a nivel geográfico hay diferencias sustanciales en materia de patrones de criminalidad, ${ }^{6}$ al menos en Chile no se había constatado la existencia y proporción de comunas cuyo número de imputados según tipos de delitos es prácticamente nulo. Esto plantea una interrogante en relación con los

\footnotetext{
${ }^{6}$ Véanse, por ejemplo, Fundación Paz Ciudadana (2008), Benavente y Melo (2006), Defensoría Penal Pública (2007), Núñez y otros (2003) y Rivera, Núñez y Villavicencio (2004).
}

\begin{tabular}{|c|c|c|c|c|c|c|c|c|c|c|c|c|c|c|}
\hline \multirow[b]{2}{*}{ No } & \multicolumn{2}{|c|}{ Robo } & \multicolumn{2}{|c|}{$\begin{array}{c}\text { Robo } \\
\text { sin violencia }\end{array}$} & \multicolumn{2}{|c|}{ Hurto } & \multicolumn{2}{|c|}{ Lesiones } & \multicolumn{2}{|c|}{ Homicidio } & \multicolumn{2}{|c|}{$\begin{array}{c}\text { Delitos } \\
\text { sexuales }\end{array}$} & \multicolumn{2}{|c|}{$\begin{array}{c}\text { Delitos } \\
\text { relacionados } \\
\text { con la droga }\end{array}$} \\
\hline & 67 & 20,0 & 32 & 10,0 & 18 & 5,0 & 10 & 3,0 & 104 & 31,0 & 44 & 13,0 & 74 & 22,0 \\
\hline Sí & 268 & 80,0 & 303 & 90,0 & 317 & 95,0 & 325 & 97,0 & 231 & 69,0 & 291 & 87,0 & 261 & 78,0 \\
\hline
\end{tabular}

Fuente: base de datos de imputados 2006, Defensoría Penal Pública (DPP). 
factores que influyen en el hecho de que una comunidad determinada tenga imputados y posibles criminales, tema que se aborda más adelante mediante la llamada ecuación de participación.

Por otra parte, seguramente debido a la falta de información detallada, en los estudios sobre el tema suele inferirse que los delincuentes habitan en el área geográfica examinada. Sin embargo, los datos a nivel de imputados permiten cuestionar este supuesto, ya que revelan que el número de ellos dependería en gran medida del tamaño de la zona en consideración. De hecho, como se aprecia en el cuadro 2, cerca de la mitad de las denuncias presentadas en una comuna determinada corresponden a personas residentes en ella. No obstante, dada la alta tasa de imputaciones dentro de las provincias y regiones, esta movilidad de los eventuales victimarios se encontraría acotada por la distancia geográfica.

En cuanto a la composición etaria de los imputados, el cuadro 3 muestra que hay una participación elevada de menores de edad, especialmente en los delitos contra la propiedad (robo, robo sin violencia y hurto).

Asimismo, los datos revelan que al parecer hay una correlación inversa entre la edad y la imputación. Como puede apreciarse en los gráficos 1 y 2, en el caso de los jóvenes el indicador de participación neta según edad - definido como el porcentaje de imputados de edad $x$ menos el porcentaje de población de esa edad- es mayor que el de otros segmentos etarios de la población. ${ }^{7}$

\footnotetext{
${ }^{7}$ Diversos autores han encontrado pruebas de que los jóvenes son más proclives a participar en actividades delictivas. Entre las razones
}

CUADRO 2

\section{Movilidad de los imputados a nive comunal, provincial y regional}

(En porcentajes)

\begin{tabular}{lccc}
\hline Tipos de delitos & Comunas & Provincias & Regiones \\
\hline Robo & 51 & 87 & 93 \\
Robo sin violencia & 58 & 88 & 93 \\
Hurto & 43 & 79 & 88 \\
Lesiones & 64 & 91 & 94 \\
Homicidio & 58 & 87 & 93 \\
Delitos sexuales & 61 & 87 & 91 \\
Delitos relacionados con & 51 & 82 & 86 \\
las drogas & & & \\
\hline
\end{tabular}

Fuente: base de datos de imputados 2006, Defensoría Penal Pública (DPP).

CUADRO 3

Delitos imputados a menores de edad (En porcentajes)

\begin{tabular}{lc}
\hline Tipos de delitos & Imputados menores de edad \\
\hline Robo & 24,03 \\
Robo sin violencia & 22,10 \\
Hurto & 11,39 \\
Lesiones & 7,97 \\
Homicidio & 10,48 \\
Delitos sexuales & 9,48 \\
Delitos de la droga & 6,36 \\
\hline
\end{tabular}

Fuente: base de datos de imputados 2006, Defensoría Penal Pública (DPP).

que explican este comportamiento se cuentan desde los aspectos psicológicos de la adolescencia hasta la percepción pesimista sobre

GRÁFICO 1

Participación neta en los delitos de robo, robo sin violencia y hurto, según edad (En porcentajes)

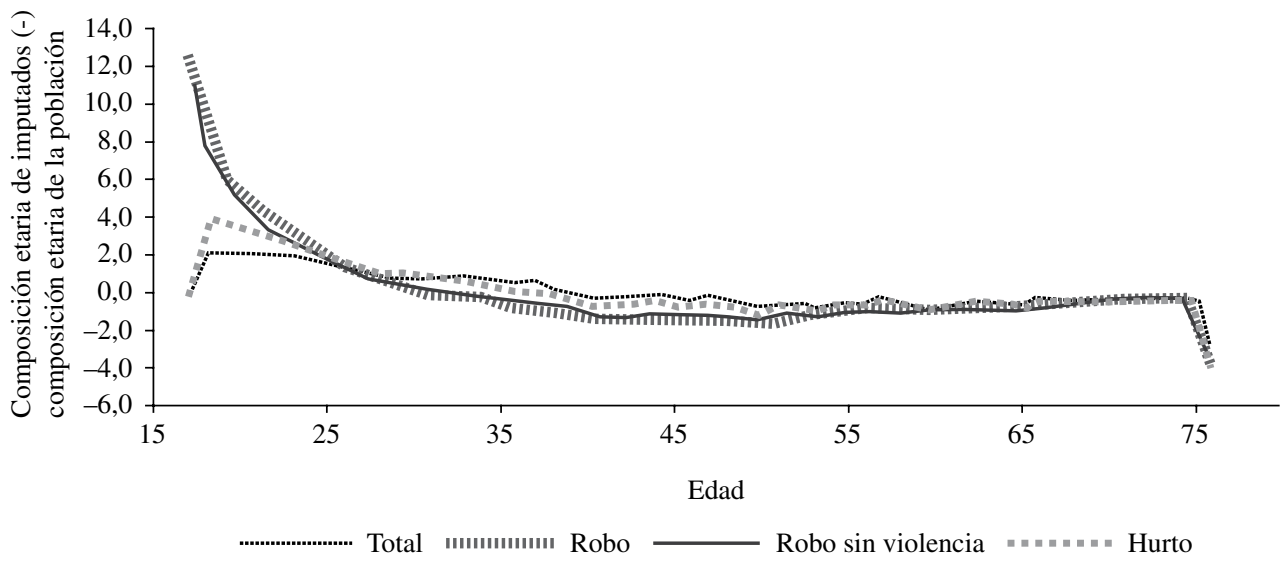

Fuente: elaboración propia sobre la base de datos de la Defensoría Penal Pública. 
En forma complementaria, del gráfico 3 se infiere preliminarmente que hay una relación inversa entre la escolaridad y el número de imputados según tipo de delito: a medida que aumenta el nivel educativo, el indicador de participación criminal - definido como el porcentaje de imputados de escolaridad $x$ menos el porcentaje de población de ese nivel de educación- disminuye. Este

el futuro en materia de ingresos legales debido a los bajos salarios que recibe este grupo etario a causa de su falta de experiencia o capacitación. Al respecto, véanse Buonanno (2003a), Freeman (1996) y Freeman (1991). hecho ha sido constatado por diversos autores (Lochner, 1999; Lochner y Moretti, 2001; Buonanno, 2003a; Buonanno, 2003b y Buonanno y Leonida, 2005), aunque en otros trabajos se encontraron resultados que prueban lo contrario (Ehrlich, 1973; Núñez y otros, 2003; Rivera, Núñez y Villavicencio, 2004). ${ }^{8}$

\footnotetext{
${ }^{8} \mathrm{Al}$ respecto, Ehrlich (1973) propuso tres posibles explicaciones: i) que la escolaridad puede aumentar el producto de las actividades ilegales, ii) que esta relación tal vez obedece a que la tasa de denuncias por parte de las víctimas de mayor educación es más elevada y iii) que las personas de mayor escolaridad son más propensas a ser victimizadas, debido a que poseen un nivel más alto de ingresos. Cabe destacar que
}

GRÁFICO 2

Participación neta en los delitos de lesiones, homicidio, sexuales y relacionados con las drogas, según edad (En porcentajes)

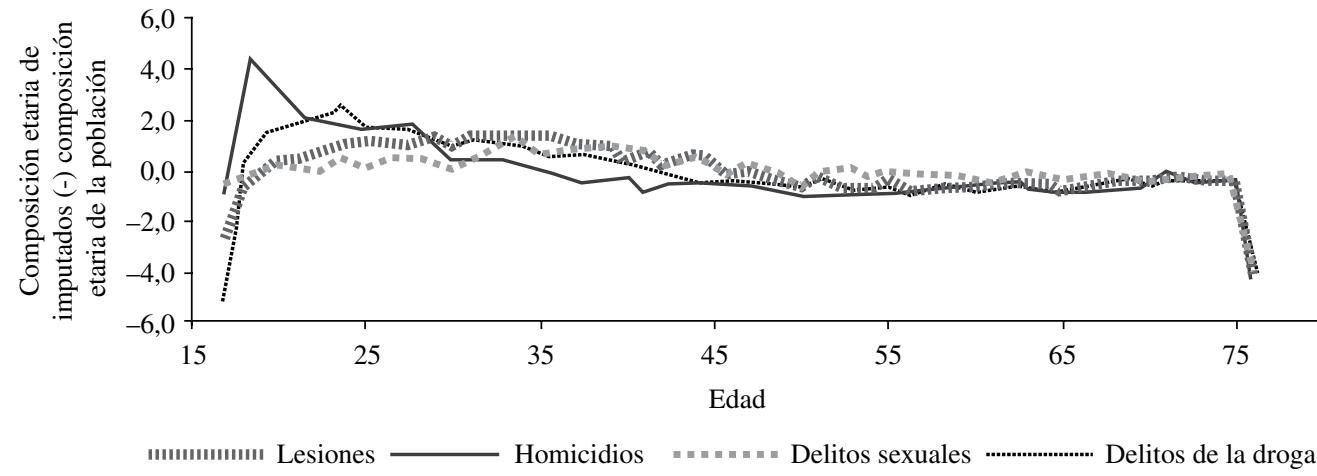

Fuente: elaboración propia sobre la base de datos de la Defensoría Penal Pública.

GRÁFICO 3

Relación entre criminalidad y nivel educacional, según tipo de delito

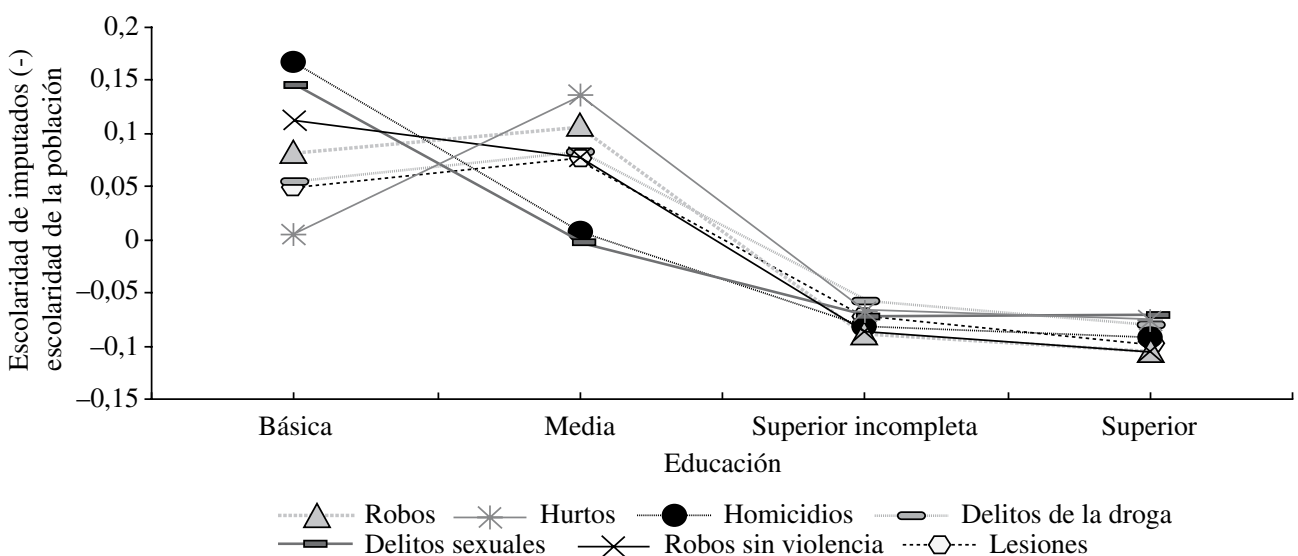

Fuente: elaboración propia sobre la base de datos de la Defensoría Penal Pública. 
Finalmente, los datos muestran que hay una correlación positiva entre el número de imputados y los distintos tipos de delitos. A partir del cuadro 4 es posible constatar que las correlaciones más elevadas corresponden a los crímenes motivados por causas económicas (robo, hurto, droga), lo cual sugiere la presencia de una serie de factores determinantes comunes que no desempeñan un rol mayormente importante en los delitos cuya motivación no es de índole económica (homicidio, delitos sexuales). En general, estos últimos poseen una baja correlación con los demás tipos de delitos, de manera que al parecer obedecen a causas diferentes que los de motivación económica.

Más adelante se hará referencia a este tema, ya que una de las conclusiones importantes de este trabajo es haber constatado que los factores condicionantes de la criminalidad difieren según el tipo de delito. Al mismo resultado se había llegado en otros estudios sobre Chile, realizados a partir de datos relacionados con las denuncias (Rivera, Núñez y Villavicencio, 2004).

CUADRO 4

Correlación entre número de imputados por cada 100.000 habitantes, según comunas y tipo de delito

\begin{tabular}{|c|c|c|c|c|c|c|c|}
\hline Delitos & Homicidios & Delitos sexuales & Delitos económicos & Lesiones & Robos & Hurtos & Delitos de la droga \\
\hline Homicidios & 1,000 & 0,372 & 0,080 & 0,193 & 0,119 & 0,137 & 0,202 \\
\hline Delitos sexuales & & 1,000 & 0,148 & 0,478 & 0,097 & 0,093 & 0,150 \\
\hline Delitos económicos & & & 1,000 & 0,403 & 0,669 & 0,650 & 0,546 \\
\hline Lesiones & & & & 1,000 & 0,295 & 0,419 & 0,312 \\
\hline Robos & & & & & 1,000 & 0,803 & 0,492 \\
\hline Hurtos & & & & & & 1,000 & 0,521 \\
\hline Delitos de la droga & & & & & & & 1,000 \\
\hline
\end{tabular}

Fuente: base de datos de imputados 2006, Defensoría Penal Pública (DPP).

Nota: Incluye las comunas que registraron imputados por los delitos señalados en 2006.

\section{III}

\section{Descripción del modelo y de los datos utilizados}

En esta sección se describe el modelo general desarrollado a fin de identificar los factores determinantes de la criminalidad. Siguiendo el modelo estático propuesto por Ehrlich (1973), se considera un individuo representativo a nivel comunal, en adelante $i=1,2, \ldots, 335$, que destina su tiempo a realizar actividades legales $\left(t_{L}^{i j}\right)$ o ilegales $\left(t_{N L}^{i j}\right)$ relacionadas con un delito determinado, en adelante $j=$ robo, robo sin violencia, hurto, lesiones, homicidio, delitos sexuales y delitos de la droga. ${ }^{9}$

las dos últimas hipótesis están vinculadas a la forma de definir la criminalidad, esto es, según las estadísticas de denuncias, lo cual no permite identificar el lugar de procedencia del victimario.

${ }^{9}$ Todos los antecedentes sobre imputados corresponden a la base de datos de imputados 2006 de la DPP, que tiene 236 delitos tipificados y agrupados en 17 categorías. El presente trabajo considera las principales categorías delictivas según su connotación social, cuya composición
A continuación, se supone que no existen costos de entrada o entrenamiento previo para realizar esas actividades ${ }^{10}$ y que los retornos pertinentes crecen de manera constante en función del tiempo destinado a ellas. No obstante, el rendimiento de las actividades ilegales es incierto, pues depende de las sanciones y de la probabilidad de captura. De este modo, dada la lógica

precisa se encuentra en Defensoría Penal Pública (2007). La base de datos de 2006 contiene un registro de 202.328 causas tratadas por la DPP. Por otra parte, los antecedentes socioeconómicos y demográficos de las comunas corresponden a la encuesta CASEN 2006.

${ }^{10}$ Este supuesto se ha discutido ampliamente, ya que implica que una persona puede transitar sin costo entre las actividades criminales y las legales. Sin embargo, con frecuencia los antecedentes penales constituyen una barrera para obtener un trabajo lícito, lo cual puede provocar una inercia en las actividades criminales. Buonanno (2003a) señala que se ha comprobado que un porcentaje muy elevado de los delincuentes realizaba actividades legales antes de dedicarse a las ilegales. 
del individuo en cuestión y considerando una función de utilidad $U($.), su problema de optimización consiste en maximizar la utilidad prevista dada por:

$$
E U\left(t_{L}^{i j}, t_{N L}^{i j}\right)=p^{i j} U\left(X_{a}^{i j}\right)+\left(1-p^{i j}\right) U\left(X_{b}^{i j}\right) \ldots
$$

sujeto a $t_{o}^{i}=t_{L}^{i j}+t_{N L}^{i j}$, donde $W_{L}^{i j}\left(t_{L}^{i j}\right)$ es el ingreso total que obtiene al destinar $t_{L}^{i j}$ unidades de tiempo a actividades legales y $W_{N L}^{i j}\left(t_{N L}^{i j}\right)$ el ingreso ilegal correspondiente; $X_{a}^{i j}=W_{L}^{i j}\left(t_{L}^{i j}\right)+W_{N L}^{i j}\left(t_{N L}^{i j}\right)-F_{N L}^{i j}\left(t_{N L}^{i j}\right)$ es el ingreso total que recibe el individuo si es capturado, lo que ocurre con una probabilidad $p^{i j}$ en que se le castiga con $F_{N L}^{i j}$, mientras que $X_{b}^{i j}=W_{N L}^{i j}\left(t_{N L}^{i j}\right)+W_{N L}^{i j}\left(t_{N L}^{i j}\right)$ representa el ingreso al no ser capturado, situación que ocurre con una probabilidad de $1-p^{i j}$. Sobre la base de este problema de optimización, la relación entre las actividades ilegales y legales queda definida mediante la siguiente ecuación:

$$
-\frac{\frac{d W_{N L}^{i j}}{d t_{N L}^{i j}}-\frac{d W_{L}^{i j}}{d t_{L}^{i j}}}{\frac{d W_{N L}^{i j}}{d t_{N L}^{i j}}-\frac{d W_{L}^{i j}}{d t_{L}^{i j}}-\frac{d F_{N L}^{i j}}{d t_{N L}^{i j}}}=\frac{p^{i j} U^{\prime}\left(X_{a}^{i j}\right)}{\left(1-p^{i j}\right) U^{\prime}\left(X_{b}^{i j}\right)} \cdots
$$

Si la remuneración de las actividades ilícitas sujetas a la probabilidad de castigo es inferior a la de las actividades legales, la persona no destinará tiempo a las primeras. Por lo tanto, para que haya delito debe cumplirse que el ingreso marginal previsto por realizar una actividad ilegal dada menos el posible castigo por cometer el delito sea superior al ingreso marginal de desarrollar una actividad legal para que resulte observable una actividad criminal, es decir:

$$
w_{N L}^{i j}>w_{N L}^{i j}-f_{N L}^{i j} p^{i j} \ldots
$$

A los efectos de la estimación, se supone que, en primer lugar, los individuos deben decidir si delinquen o no (decisión de participación), para lo cual evalúan la ecuación 3. En caso afirmativo, toman una segunda decisión que consiste en definir el tiempo que destinarán a la actividad criminal (ecuación de imputados) de acuerdo con la ecuación 2. De esta manera, para tomar en cuenta el posible sesgo de selección que implicaría la presencia de una correlación entre ambas decisiones, las estimaciones econométricas de imputados y de participación se calcularon mediante modelos Heckit, según máxima verosimilitud, suponiendo que los errores presentan una distribución normal bivariada.

La decisión de participación, definida mediante la ecuación 3, resultará positiva si el ingreso ilegal neto de los posibles castigos supera el ingreso legal. Sin embargo, dado que no se dispone de información sobre el ingreso ilegal percibido por los agentes, y mucho menos de los devengados por concepto de delitos tipo $j$, se aproximó dicha variable mediante el ingreso per cápita $Y_{r e g}^{i}$ de la región a la cual pertenece la comuna de origen del imputado. Esta aproximación es factible debido a que las oportunidades de obtener ingresos ilegales están relacionadas con la riqueza de que podrían disponer las víctimas, las cuales — como se mostró en la sección anterior- pertenecen mayoritariamente a la región que circunscribe su propia comuna. De esta manera, se tiene que:

$$
w_{N L}^{i j}=w_{N L}^{i j}\left(Y_{r e g}^{i}\right)=X_{w_{N L}}^{i} \alpha_{1}^{j} \ldots
$$

donde $X_{w_{N L}}^{i}=\left[Y_{r e g}^{i}\right]$

El ingreso legal se aproximó según el ingreso medio de la comuna $\left(Y_{\text {comunal }}^{i}\right)$; sin embargo, a fin de captar las posibles disparidades en su distribución, se incluyó como variable adicional el porcentaje de pobres de dicha comuna $\left(\right.$ pobres $\left.^{i}\right) .{ }^{11}$ En el modelo se consideró la variable desempleo de la población entre 18 y 40 años $\left(\right.$ desempleo $^{i}$ ) para incluir las posibilidades y oportunidades reales de obtener ingresos legales. ${ }^{12}$ Dadas las consideraciones antes señaladas, el ingreso legal queda definido de la siguiente manera:

$w_{L}^{i j}=w_{L}^{i j}\left(Y_{\text {comunal }}^{i}\right.$ pobres $^{i}$, desempleo $\left.^{i}\right)=X_{w_{L}}^{i} \alpha_{2}^{j}$...

donde $X_{w_{L}}^{i}=\left[Y_{\text {comunal }}^{i}\right.$ pobres $^{i}$, desempleo $\left.^{i}\right]$.

Por otra parte, se supone que la función de castigos $f_{i j}$ equivale a la pena que la ley impone a los delitos tipo $j$,

\footnotetext{
${ }^{11}$ Fajnzylber, Lederman y Loayza (2002) estudiaron la criminalidad agregada de diversos países y encontraron significativa la influencia de la disparidad de ingresos, motivo por el cual se consideró importante incluirla.

${ }^{12}$ Sin embargo, como se indicó anteriormente, se ha comprobado que un porcentaje mayoritario de los individuos que delinquen dispone de empleo. Imrohoroglu, Merlo y Rupert (2001) estimaron que en los Estados Unidos alrededor del $70 \%$ de los criminales tenía empleo al momento de cometer un delito.
} 
esto es $\left(c_{\text {ley }}^{j}\right)$, que sería la misma en todas las comunas según el delito de que se trate. Para los propósitos de nuestra estimación, sus efectos no podrán determinarse, ya que estarán incluidos en el término constante de las ecuaciones.

De conformidad con las críticas de Block y Heineke (1975) y William y Sickles (2002) (véase además Buonanno, 2003c), se incluyeron las siguientes variables sociodemográficas de que dependería la criminalidad y que se emplean usualmente en los estudios sobre el tema: i) porcentaje de población entre 13 y 17 años $\left(p o b_{13-17}^{i}\right)$, ii) porcentaje de población entre 18 y 40 años $\left(p o b_{18-40}^{i}\right)$, iii) porcentaje de hogares monoparentales cuyo jefe de hogar es la madre ( hog $\left._{\text {mujer }}^{i}\right)$, iv) porcentaje de hogares con menores entre 13 y 18 años, en que ambos padres trabajan ${ }^{13} \mathrm{y}$, finalmente, $v$ ) años de escolaridad media de los mayores de 13 años de la comuna $\left(E d u c^{i}\right)$.

Dado lo anterior, el castigo que se aplica en la comuna $i$ por los delitos tipo $j$ puede expresarse mediante la siguiente ecuación:

$$
f^{i j}=f^{i j}\left(c_{\text {ley }}^{j}, C S\left(\operatorname{pob}_{13-17}^{i}, \operatorname{pob}_{18-40}^{i}, \text { hog }_{\text {mujer }}^{i}, \text { hog }_{\text {padres-trabaj }}^{i}, E d u c^{i}\right)\right)=X_{f}^{i} \alpha_{3}^{j} \ldots
$$

donde $X_{f}^{i}=\left[\overline{c_{\text {ley }}^{j}}, p o b_{13-17}^{i}, p o b_{18-40}^{i}, \operatorname{hog}_{\text {mujer }}^{i}, \operatorname{hog}_{\text {padres-trabaj }}^{i}, E d u c^{i}\right]$ y $\overline{c_{\text {ley }}^{j}}$ la constante ya mencionada.

Por otra parte, y siguiendo las hipótesis planteadas en Becker (1968), la variable $p^{i j}$ que representa la posibilidad de ser castigado depende de la probabilidad de captura

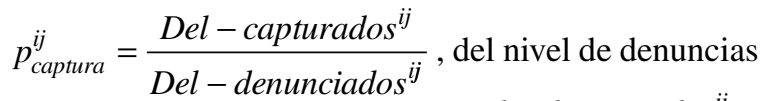

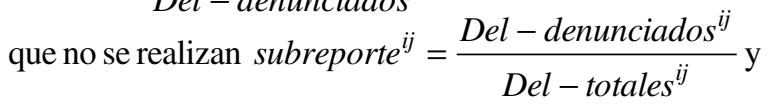
de la posibilidad de ser encontrado culpable, condicional a ser capturado $p_{\text {responsabilidad }}^{i j}=\frac{\text { Del }- \text { castigados }^{i j}}{\text { Del }- \text { capturados }^{i j}}$.

A los efectos de la estimación, se supuso que la probabilidad de captura es una función del número de comisarías a nivel comunal $p_{\text {captura }}^{i j}=p_{\text {captura }}^{i j}\left(\text { comisaria }^{i}\right)^{14}$ y que el número de delitos que no se denuncian en las distintas comunas es constante. La probabilidad de ser castigado una vez capturado $\left(p_{\text {responsabilidad }}^{i j}\right)$ se estimó a partir del cociente entre los imputados que se declaran culpables y el total de imputados a nivel regional, ambos en el año 2005. ${ }^{15}$ Por lo tanto, la probabilidad

\footnotetext{
${ }^{13}$ William y Sickles (2002) muestran que el entorno familiar y vecinal desempeña un papel importante en el comportamiento criminal. ${ }^{14}$ Los antecedentes policiales corresponden a las estadísticas oficiales de Carabineros de Chile que se publican en la página web del Instituto Nacional de Estadísticas de Chile, sección estadísticas policiales [en línea] www.ine.cl.

${ }^{15}$ En rigor, dada la falta de información sobre 2005 a nivel nacional, la variable se construyó con los datos del segundo semestre de este año. Se utilizó el dato rezagado en el supuesto de que este puede ser observado por los individuos en el período siguiente.
}

de ser castigado queda representada por la siguiente ecuación:

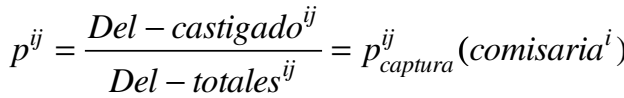

$$
\begin{aligned}
& \times \text { subreporte }^{i j} \times p_{\text {responsabilidad }}^{i j}=X_{p}^{i} \alpha_{4} \ldots
\end{aligned}
$$

donde $X_{p}^{i}=\left[\right.$ comisaria $\left.^{i}, \overline{\text { subreporte }}, p_{\text {responsabilidad }}^{i j}\right] \mathrm{y}$ subreporte es la constante indicada.

Al reescribir y agregar un término aleatorio de perturbación, la ecuación 3 del modelo de participación se expresa de la siguiente manera:

$$
\begin{aligned}
& S^{i j}=w_{N L}^{i j}(.)-f^{i j}(.) p^{i j}-w_{L}^{i j}(.) \\
& +\varepsilon_{\text {participación }}^{i}=X^{i} \Gamma^{j}+\varepsilon_{\text {participación }}^{i}
\end{aligned}
$$

donde $\Gamma=\left[\alpha_{1}, \alpha_{2}, \alpha_{3}, \alpha_{4}\right]$ y $X^{i}=\left[X_{W_{N L}}^{i}, X_{W_{L}}^{i}, X_{f}^{i}, X_{p}^{i}\right]$.

Cabe destacar que, en la ecuación anterior, una comuna $i$ presentará tasas de imputación positivas siempre y cuando $S^{i j}>0$. Genéricamente, en el caso de cualquier tipo de delito, definamos la variable dicotómica $H^{i}$ cuyo valor es 1 si la comuna $i$ presenta imputaciones por el delito analizado y 0 en caso contrario. La ecuación de participación del delito de que se trate puede estimarse mediante un modelo probit, suponiendo que la variable $\varepsilon_{\text {part }}^{i}$ es normal, con media 0 y varianza $\sigma_{\varepsilon_{\text {participacion }}^{i}}$ :

$\operatorname{Prob}\left(H^{i}=1\right)=\operatorname{Pr} o b\left(\varepsilon_{\text {part }}^{i}>-X^{i} \Gamma\right)=\Phi\left(\frac{X^{i} \Gamma}{\sigma_{\varepsilon_{\text {participación }}}}\right)$ 
En cuanto a los imputados, los niveles de actividad criminal se determinan mediante la ecuación 2, así como por las restricciones de tiempo de que dispone el individuo representativo. Esta ecuación muestra que el número de imputados por cada tipo de delito es una función de las mismas variables que la ecuación de participación. Sin embargo, a los efectos de la estimación se consideró apropiado expresar el número de imputados en forma logarítmica mediante la ecuación 10 , cuyas variables están relacionadas linealmente más un término aleatorio. Asimismo, a fin de identificar en forma adecuada la ecuación de participación, se excluyeron algunas variables de la ecuación de imputados - tema que se discute en la próxima sección-, de modo que esta se expresó de la siguiente manera:

$$
\operatorname{Ln}\left(t_{N L}^{i j}\right)=\Pi^{j} X^{i}+\varepsilon_{o}^{i} \ldots
$$

donde $\Pi^{j}=\left[\pi_{1}, \pi_{2}, \pi_{3}, \pi_{4}\right]$. No obstante, dado que la criminalidad solo es observable cuando $S^{i j}$ es mayor que 0 , la existencia de una posible correlación entre los términos aleatorio $\left(\varepsilon_{o}^{i}\right)$ y de error de la ecuación de participación impide que el término de error condicional sea igual a 0 , lo que contribuye a sesgar la estimación según mínimos cuadrados. De esta manera, a fin de controlar las eventuales diferencias idiosincrásicas entre las comunas se incorporaron las siguientes variables, ${ }^{16}$ que también se incluyeron en la ecuación de participación: i) la variable dicotómica peq-localidad ${ }^{i}$, que toma valor 1 si la comuna $i$ tiene menos de 7.000 habitantes;

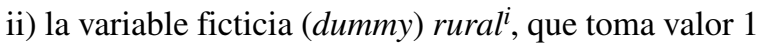
si la población rural de la comuna $i$ es superior al 50\%; iii) Densidad ${ }^{i}$, que representa el número de habitantes por kilómetro cuadrado de la comuna $i$; iv) la variable dicotómica norte, que toma valor 1 si la comuna $i$ pertenece a las regiones I, II, III y IV, y v) centro, que toma valor 1 si la comuna $i$ pertenece a las regiones $\mathrm{V}$, VI y Metropolitana. Además, en la ecuación de participación se incluyó la variable distancia ${ }^{i}$, que mide la distancia entre la comuna $i$ y el principal centro urbano de la región pertinente.

\footnotetext{
${ }^{16}$ En diversos estudios se ha demostrado que las localidades pequeñas tienden a presentar tasas inferiores de criminalidad, dado que a los delincuentes les resultaría más difícil pasar desapercibidos (Rivera, Núñez y Villavicencio, 2004; Glaeser y Sacerdote, 1999).
}

Siguiendo a Sah (1991), también se incluyó el número de imputados de la provincia, expresado en logaritmos (Lndelprov), bajo la hipótesis de que las zonas en que existe un entorno de mayor criminalidad podrían reflejar una baja probabilidad de captura y que, por lo tanto, la población de las comunas pertinentes actualizará sus expectativas y presentará una propensión más significativa a realizar actividades ilícitas. De este modo, la ecuación final a estimar queda expresada de la siguiente manera:

$$
\begin{aligned}
& E\left(\operatorname{Ln}\left(t_{N L}^{i j}\right) \mid S^{i j}>0\right)=\Pi^{j} X^{i}+ \\
& \text { Idiosincráticas }+\mathrm{E}\left(\varepsilon_{\mathrm{o}}^{\mathrm{i}} \mid S^{i j}>0\right) \ldots
\end{aligned}
$$

Suponiendo que los términos de error de ambas ecuaciones $\left(\varepsilon_{o}^{i}\right.$ y $\left.\varepsilon_{\text {participación }}^{i}\right)$ provienen de una distribución normal de medias 0 , con varianzas $\sigma_{\varepsilon_{o}^{i}} \sigma_{\varepsilon_{p}^{i}}$

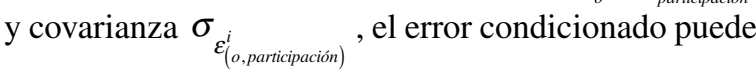
calcularse mediante la siguiente ecuación:

$$
E\left(\varepsilon_{o}^{i} \mid S^{i j}>0\right)=\rho \sigma_{\varepsilon_{o}^{i}} \lambda\left(\frac{\Gamma X^{i}}{\sigma_{\varepsilon_{\text {participación }}^{i}}}\right) \cdots
$$

donde $\rho$ es el coeficiente de correlación de las ecuaciones de participación y de imputados, mientras que $\lambda(\cdot)$ es la inversa de la razón de Mills. La estimación de los coeficientes de ambas ecuaciones se realizó mediante el método de máxima verosimilitud a fin de asegurar la consistencia de los estimadores. ${ }^{17}$

\footnotetext{
${ }^{17}$ Solo a modo explicativo, cabe señalar que el análisis no se desarrolló a partir de la estimación de un panel desbalanceado, por los siguientes motivos: i) la ausencia de información anterior a 2006 sobre la procedencia comunal de un gran número de imputados; ii) la falta de datos anuales sobre las variables explicativas durante el período relevante, dado que estos corresponden a las encuestas CASEN que se realizan aproximadamente cada dos años, y iii) la posible subestimación de los imputados en algunas comunas, debido al desplazamiento de los delincuentes potenciales desde su región de origen hacia zonas en que no se había puesto en marcha la reforma procesal penal. Recordemos que la implementación de esta fue gradual: la última región en incorporarse al nuevo sistema fue la Región Metropolitana (Santiago), en el año 2005. Para mayor información al respecto, véase Defensoría Penal Pública (2007).
} 


\section{IV}

\section{Resultados del modelo}

Dado que la ecuación de imputados según tipo de delito es la que ofrece los resultados de mayor interés, a continuación se presentan y comentan los principales alcances de ella (los resultados de las demás estimaciones se detallan en el anexo 2).

Los resultados en cuestión se obtuvieron a partir de dos especificaciones econométricas para cada tipo de delito: una que incluyó todas las variables presentadas anteriormente y otra en que solo se consideraron las variables significativas al 90\% (modelo reducido), pero conservando las variables económicas más importantes $\left(Y_{\text {comunal }}^{i}, Y_{\text {reg }}^{i}\right.$ y $\left.p_{\text {responsabilidad }}^{i j}\right)$, independientemente de su significancia estadística.

No obstante lo anterior, si bien el análisis teórico desarrollado indica que los factores determinantes de la participación en actos delictivos son los mismos que explican el nivel de criminalidad (ecuación de imputados), la variable distancia se incluyó solamente en la ecuación de participación a fin de identificarla en forma correcta (estimación de Heckman). Con el mismo propósito, en los modelos reducidos se omitieron otras variables de la ecuación de imputados para mejorar la identificación de las ecuaciones. ${ }^{18}$

Mediante la prueba de independencia entre la ecuación de imputados y la ecuación de participación — que fue la de Wald, al 90\% — no fue posible rechazar la hipótesis de independencia, salvo en el caso del homicidio y de los delitos sexuales, lo cual equivale a señalar que la ecuación de imputados podría estimarse sobre la base del nivel de criminalidad observado. Los resultados de ambas ecuaciones se presentan en el anexo. ${ }^{19}$

A fin de validar el resultado anterior, se analizó la correlación entre la razón de Mills y las variables explicativas de la ecuación de imputados, para descartar las correlaciones elevadas que pudieran afectar la validez

\footnotetext{
${ }^{18}$ Las variables omitidas a los efectos de identificación en las ecuaciones de participación de los distintos delitos fueron las siguientes: en robo: distancia, centro, peq-localidad, rural y pobres; en robo sin violencia: distancia, pob 13-17, pobres, hog-padres-trabajo y desempleo; en hurto: distancia y educ; en homicidios: distancia, pob 18-40, norte y educ; en delitos sexuales: distancia, pob 13-17, rural y desempleo, y en delitos tipificados en la ley de drogas: distancia, norte, peqlocalidad y desempleo.

${ }^{19}$ El delito de lesiones no fue estudiado en la ecuación de participación, dado que está presente en el $97 \%$ de las comunas analizadas.
}

de la prueba y la consistencia de los coeficientes estimados. De acuerdo con lo informado en el anexo 2, se encontraron $\mathrm{R}^{2}$ inferiores al $57 \%$ en todos los modelos reducidos, lo que indicaría que la correlación entre la razón de Mills y las variables explicativas de la ecuación de imputados es reducida. ${ }^{20}$

Los resultados de la ecuación de imputados según tipo de delito podrían ser interesantes. En primer lugar, indican que si bien hay algunos factores determinantes que son transversales, existe un grado importante de heterogeneidad entre los imputados por los distintos delitos, lo que señala la presencia de motivaciones y causas diferentes.

Por otra parte, el ingreso comunal muestra una relación inversamente proporcional a la criminalidad, con un coeficiente significativo al $99 \%$ en todos los delitos - salvo el homicidio que, sin embargo, conserva el signo previsto- Este resultado concuerda con las predicciones del modelo teórico e indica que las comunas más pobres son más propensas a registrar imputados por una amplia gama de delitos. Las elasticidades asociadas al ingreso comunal (véase el anexo 2) varían desde $-0,25$ en el caso de las lesiones hasta $-0,65$ en el del robo sin violencia.

En el caso de los delitos cuya motivación es claramente de índole económica (robo, robo sin violencia y hurto), la relación entre el ingreso regional y el número de imputados es positiva y significativa, lo que difiere de otros delitos en que, eventualmente, el móvil no es de carácter económico. Esto último concuerda con las hipótesis sobre las oportunidades de ingreso ilegal que representarían los delitos motivados por circunstancias de índole económica. De acuerdo con el resultado de las estimaciones, el rango de elasticidades vinculadas a estos últimos varía entre 0,52 (hurto) y 0,97 (robo sin violencia).

Los efectos de la variable disuasión solo son significativos en los delitos relacionados con la ley de drogas. El desempleo comunal es estadísticamente significativo solo en el caso del robo, lo que concuerda con el modelo

\footnotetext{
${ }^{20}$ El procedimiento desarrollado es similar al de Elias y Okseniuk (2002), quienes aplicaron la recomendación de Nawata y Nagase (1996). Al respecto, véase [en línea] http://www.aaep.org.ar/espa/ anales/PDF_02/elias_okseniuk.pdf.
} 
teórico, y su elasticidad es igual a 0,23. Por otra parte, hay una relación positiva entre la educación y el número de imputados por los delitos de robo y hurto, resultado que corrobora lo encontrado en otros estudios sobre Chile y a nivel mundial.

En las comunas de la zona norte de Chile hay mayor propensión a registrar imputados por una amplia gama de delitos, lo que concuerda con los resultados de trabajos anteriores (Núñez y otros, 2003; Rivera, Núñez y Villavicencio, 2004).

La proporción de jóvenes de las comunas no afecta significativamente el número de imputados según tipo de delitos, excepto en el caso de lesiones, cuya elasticidad

\section{$\mathrm{V}$}

\section{Conclusiones}

Este trabajo constituye un esfuerzo por examinar los factores determinantes de la criminalidad desde la perspectiva de la localidad de origen de los imputados, en vez de seguir el enfoque tradicional de emplear datos basados en las denuncias de la población y de considerar el lugar en que estas se registraron como aquel de procedencia del imputado. De esta manera, en el presente trabajo se admite la disociación geográfica entre la localidad a la que pertenecen las víctimas y los victimarios, con lo cual las características del lugar de residencia de las primeras se convierten en un factor determinante de la propensión a delinquir (ingresos ilegales).

Al respecto, en este trabajo se muestra que cerca de la mitad de las denuncias registradas se realiza fuera de la comuna de residencia del victimario, aunque la gran mayoría de ellas corresponde a la misma región, lo cual indica que sería inadecuado analizar la criminalidad sobre la base de los datos relacionados con las denuncias a nivel comunal. Por otra parte, los estudios basados en las imputaciones a nivel regional podrían verse limitados si la heterogeneidad social y económica dentro de las regiones es elevada, como en el caso de Chile y de los demás países de América Latina.

Otro resultado destacable de este trabajo es la elevada correlación que existe entre las comunas de los imputados por distintos tipos de delitos, lo cual plantea la interrogante de cuáles serían los factores determinantes de la propensión de estas a registrar imputados. Al respecto, en este estudio se señala que si bien las causas de los actos delictivos son diferentes, hay algunas que son es de 0,48. Las comunas rurales tienden a presentar menos imputados por diversos delitos. Por último, la dotación de comisarías de la comuna tiene un efecto importante y significativo en el número de imputados por todos los delitos, con excepción del homicidio, aunque el signo es también positivo. La interpretación de este resultado es ambigua: por una parte, tal vez refleja el éxito de los esfuerzos policiales por aprehender a los delincuentes, pero también podría obedecer al hecho de que las comisarías se ubican de preferencia en las comunas cuya población es más propensa a cometer delitos. Lo que en definitiva explica el signo es todavía un problema abierto. transversales. En particular, y todo lo demás constante, el número de imputados tiende a ser superior en las comunas más pobres, pertenecientes a zonas urbanas, de la zona norte del país y de mayor escolaridad. Este último resultado se ha constatado en otros estudios sobre Chile y el mundo, aunque aún existe un debate en torno a su interpretación.

Por otra parte, el desempleo no influye mayormente en el número de imputados y la variable disuasión - la probabilidad de ser capturado que había dentro de una comuna determinada en períodos anteriores - no afecta de manera significativa las imputaciones. La presencia policial, medida según la dotación de comisarías de la comuna, incrementa marcadamente el número de imputados de las diversas comunas en casi todos los tipos de delitos.

Otro resultado de interés es que las oportunidades de obtener ingresos ilegales, aproximadas según el ingreso regional, contribuyen a aumentar el número de imputados de cada comuna solo en el caso de los delitos en que estos son relevantes, es decir, aquellos de motivación principalmente económica (robo, robo sin violencia y hurto), y no en el de otros tipos de delitos (homicidio, lesiones, delitos sexuales). Estos resultados concuerdan con los encontrados en estudios anteriores sobre Chile ${ }^{21}$ y sugieren que, en los delitos

\footnotetext{
${ }^{21}$ Véanse por ejemplo Rivera, Núñez y Villavicencio (2004) y Núñez y otros (2003), donde también se observa que los factores económicos desempeñan un rol importante, principalmente en los delitos de motivación económica.
} 
que no obedecen a circunstancias de índole económica, es necesario avanzar en la búsqueda de explicaciones y factores determinantes desde otros enfoques y disciplinas, tales como la sociología y la psicología.

Existen numerosos ámbitos de investigación futura sobre los factores determinantes de la criminalidad desde el punto de vista del lugar de procedencia de imputados y victimarios. En particular, convendría estudiar el grado de persistencia temporal de la delincuencia, tema que no fue posible abordar en este trabajo por tratarse de un análisis de corte transversal. Además, sería interesante diferenciar entre quienes son imputados por primera vez y quienes lo son repetidamente, ya que se supone que podrían verse involucrados en una carrera delictiva. Asimismo, es posible analizar una amplia gama de causas determinantes de la criminalidad a nivel regional que poseen adecuado sustento conceptual o teórico, más allá de las incorporadas en este trabajo.

Por último, cabe agregar que el hecho de no rechazar la hipótesis de independencia entre las ecuaciones de participación y de selección no obedece necesariamente a los eventuales problemas de identificación de esta última. Aunque la decisión de participar dependería de variables socioeconómicas, las pruebas empíricas indican que ella no estaría vinculada al nivel de criminalidad, contrariamente a lo que sugiere la aproximación teórica desarrollada en este trabajo.

ANEXO 1

Relaciones entre criminalidad e imputabilidad

Idealmente, el indicador de imputados de un espacio geográfico dado corresponde a la razón entre el porcentaje de personas que han participado en actividades criminales y el de las residentes en dicha zona. No obstante, uno de los problemas del análisis de la criminalidad es la existencia de componentes que no son observables y que impiden utilizar directamente esta aproximación.

Como se dijo en la introducción, en los estudios sobre la criminalidad generalmente se utilizan las bases de datos de las denuncias, de manera que no puede determinarse el lugar de procedencia de los imputados. Otro problema de esta aproximación es el número de delitos que no se denuncian, lo cual se intentó eliminar mediante las técnicas de datos de panel o suponiendo que es aleatorio.

Si bien el enfoque aplicado en el presente documento se libera del primer problema señalado, comparte el de las denuncias no realizadas, dado que una persona no puede ser imputada por un delito determinado sin la denuncia pertinente de la víctima o del Ministerio Público. No obstante, además de lo señalado en el párrafo anterior, el uso de la razón de imputados es objetable desde el punto de vista jurídico, en el sentido de que los imputados serían implícitamente responsables de los delitos, sin que ello haya sido determinado por un juez, y de que existe la posibilidad de que una persona inocente sea aprehendida, declarada culpable o ambos.

Sin embargo, como puede apreciarse en la ecuación 1A, la utilización de la razón de imputados es válida si la relación $\frac{C r i_{d e n u c-n o-c a p t}-I n o c_{\text {capt }}}{P o b}$ es aleatoria o puede captarse mediante alguna de las variables observables, tales como el esfuerzo policial o las características socioeconómicas. En el presente estudio se consideró que este factor no depende de causas de índole socioeconómica, de manera que se procedió a correlacionar el grado de responsabilidad criminal con el nivel de ingreso per cápita, encontrándose valores muy bajos. No obstante, el efecto de la presencia policial se controló incluyendo una variable ficticia que identificaba la existencia de una comisaría en la comuna analizada.

$$
\begin{aligned}
& \frac{C r i}{P o b}=\frac{C r i_{d e n u c}+C r i_{n o-d e n u c}}{P o b}=\frac{C r i_{d e n u c-c a p t}+C r i_{d e n u c-n o-c a p t}+C r i_{n o-d e n u c}}{P o b} \\
& =\frac{C r i_{d e n u c-c a p t}+C r i_{d e n u c-n o-c a p t}+\text { Inoc }_{\text {capt }}-\text { Inoc }_{\text {capt }}+C r i_{\text {no-denuc }}}{\text { Pob }} \\
& =\frac{\text { Imputad }}{P o b}+\frac{\text { Cri }_{\text {denuc-no-capt }}-\text { Inoc }_{\text {capt }}}{P o b}+\frac{C r i_{\text {no-denuc }}}{P o b}
\end{aligned}
$$

donde:

$C r i=$ número de personas involucradas en una actividad criminal determinada.

$C r i_{\text {denuc }}=$ número de personas involucradas en una actividad criminal que presentan denuncias, sea identificados o no.

$\mathrm{Cri}_{\text {no-denuc }}=$ número de personas involucradas en una actividad criminal que no presentan denuncias.

$\mathrm{Cri}_{\text {denuccapt }}^{\text {no-denuc }}=$ número de criminales denunciados y capturados.

$\mathrm{Cri}_{\text {denuc-no-capt }}=$ número de criminales denunciados y no capturados.

Inoc $_{\text {capt }}=$ número de imputados que, siendo inocentes, fueron capturados. 


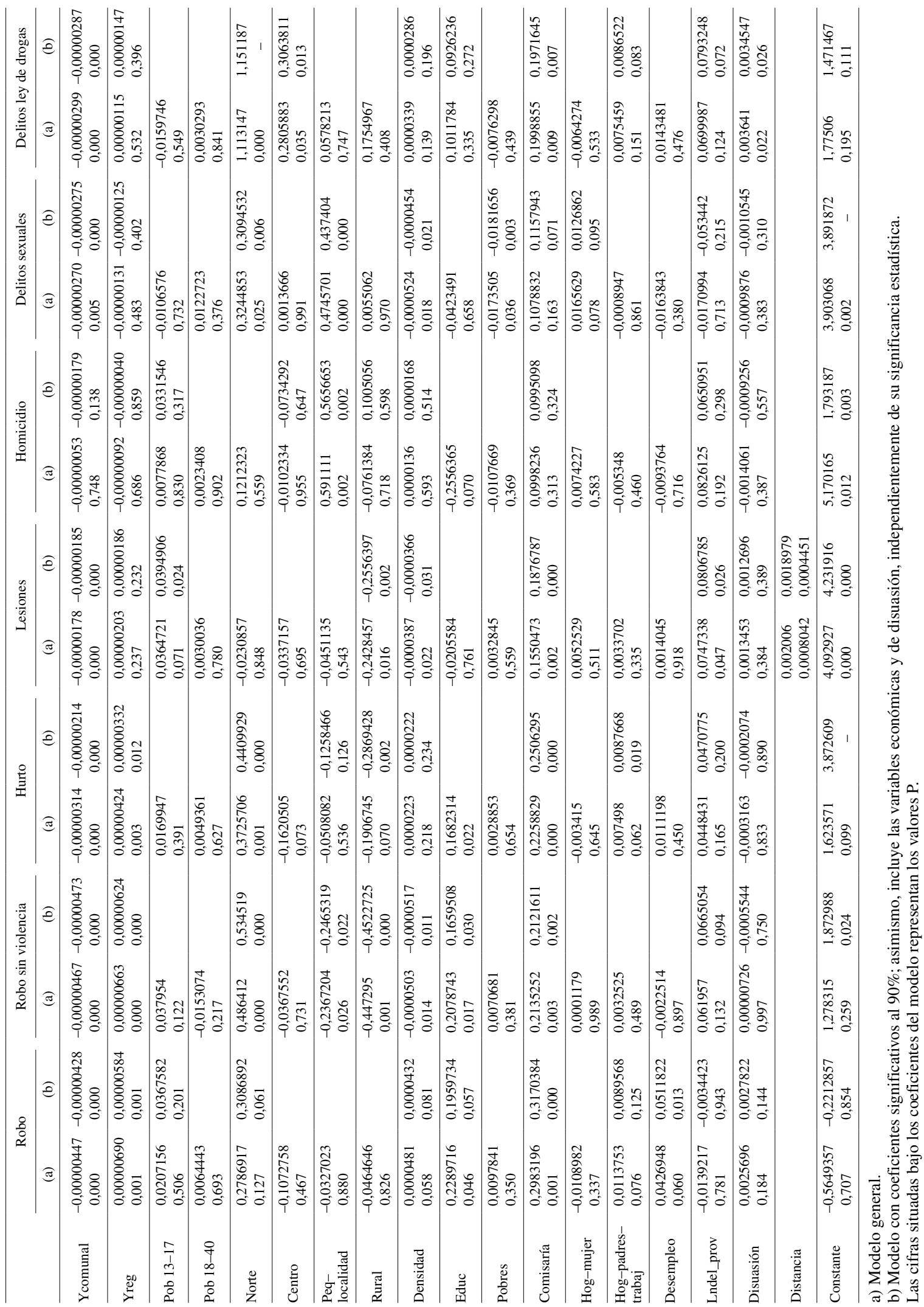




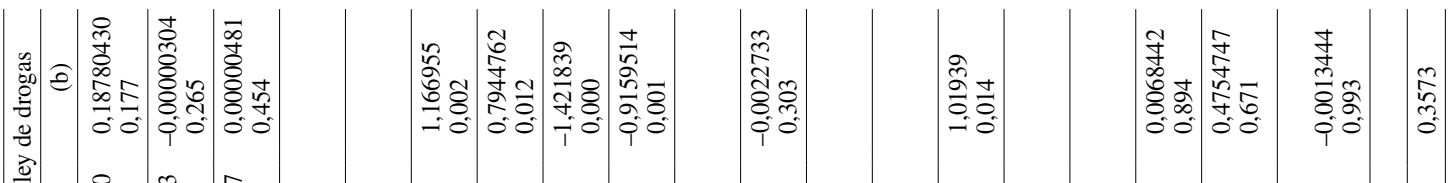

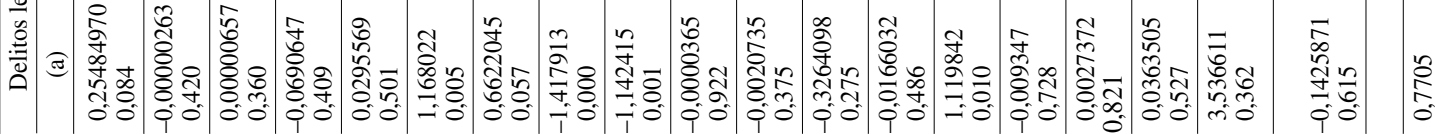

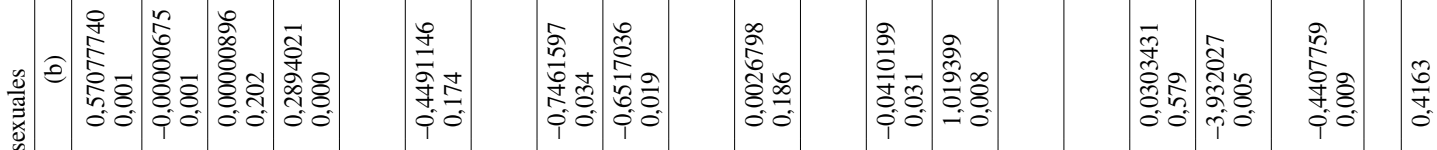

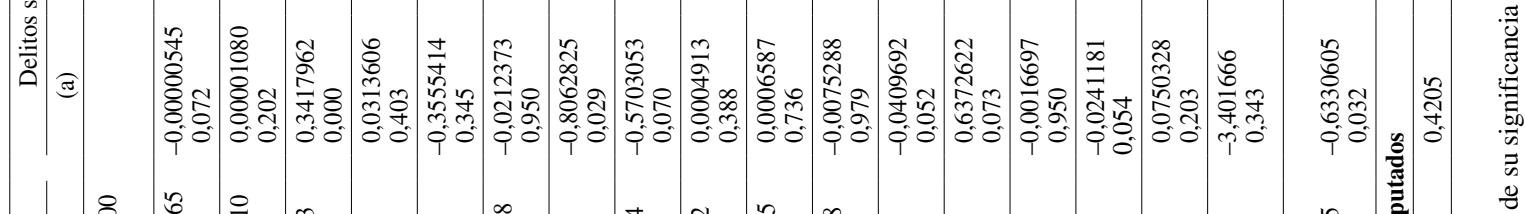

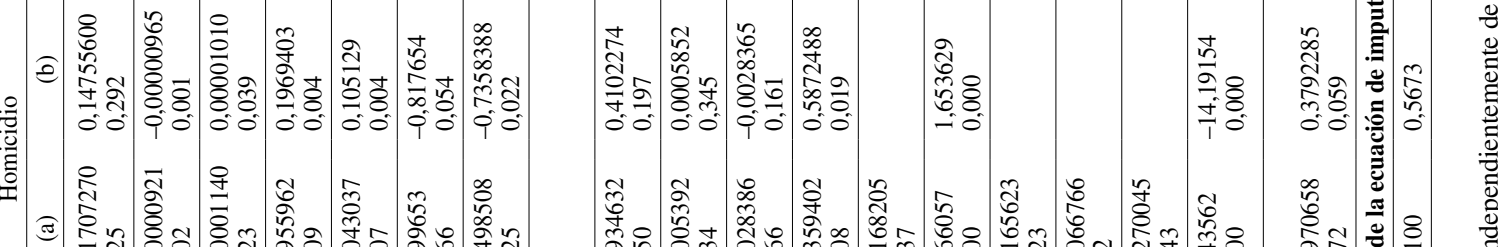

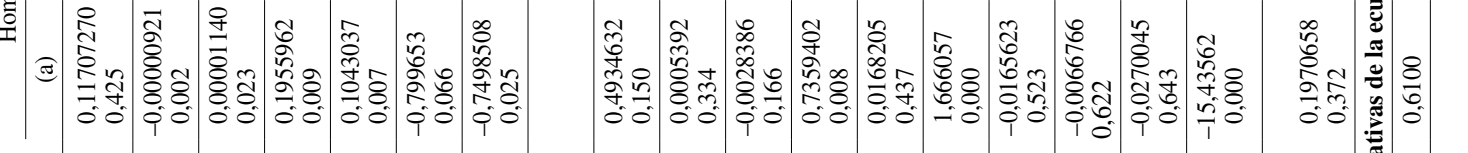

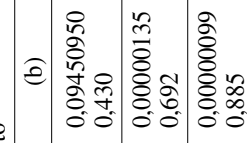

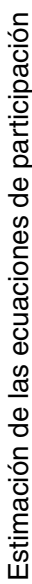

竞

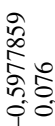

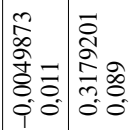

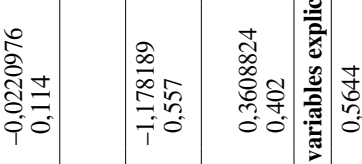

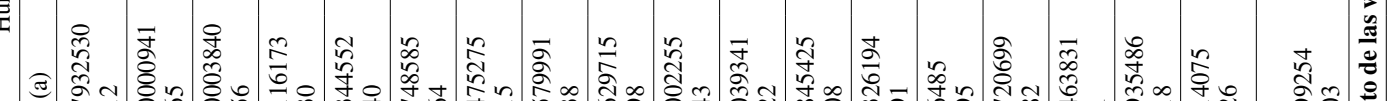

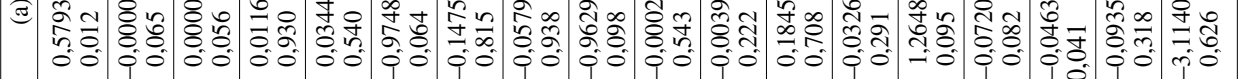

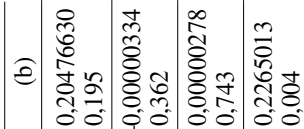

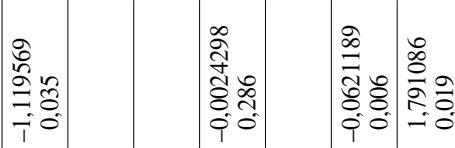

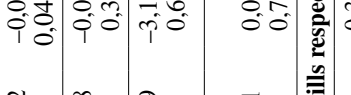

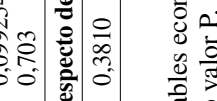
竞 (a) $\frac{0}{0}$

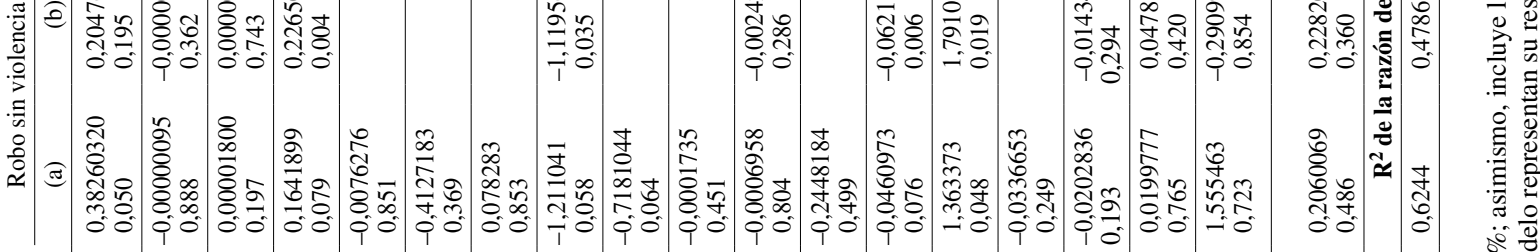
등

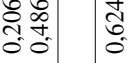




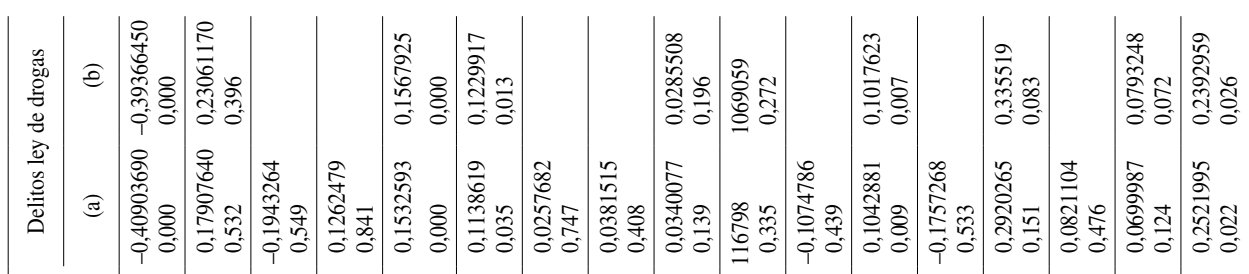

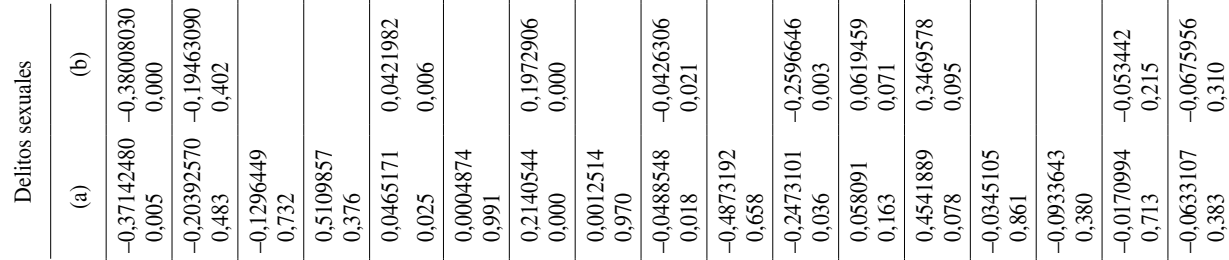

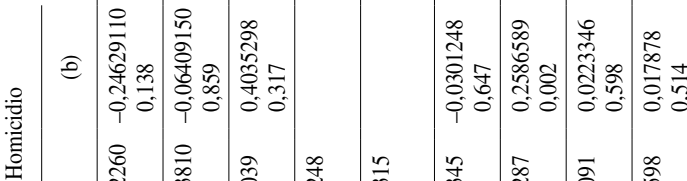

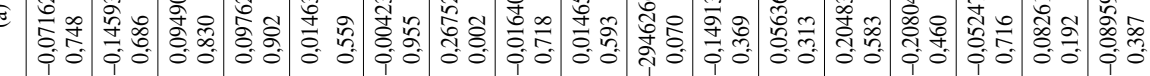

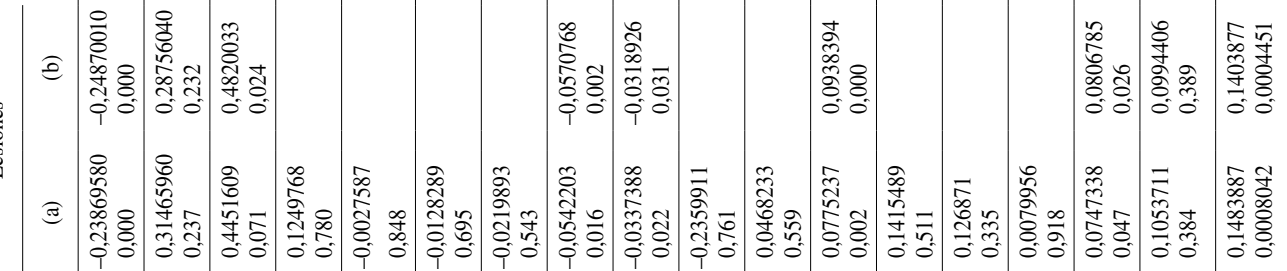

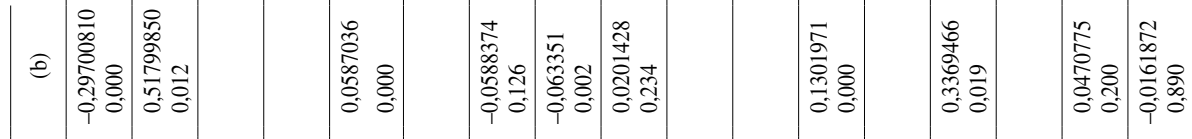

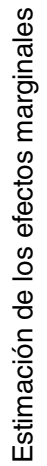
善

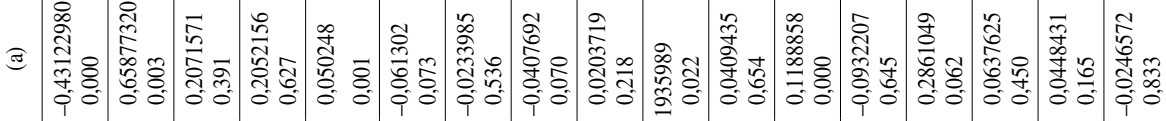

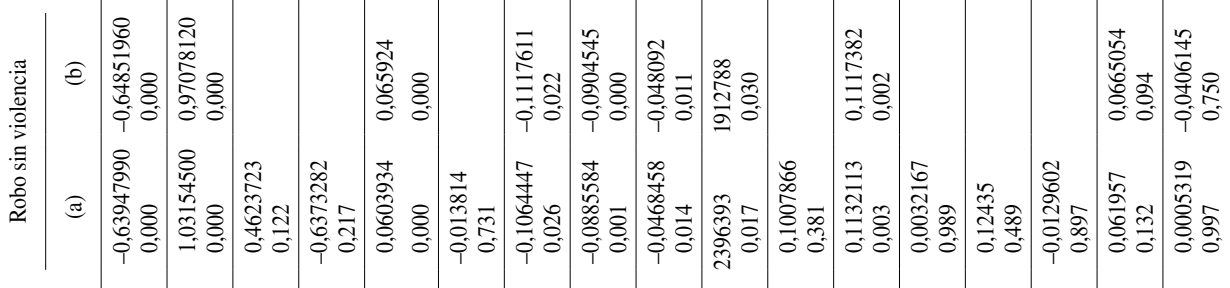

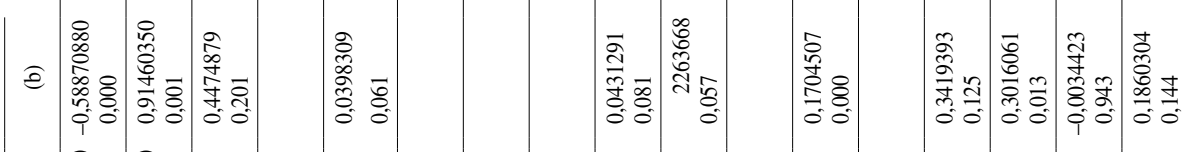
$\stackrel{\circ}{\circ}$

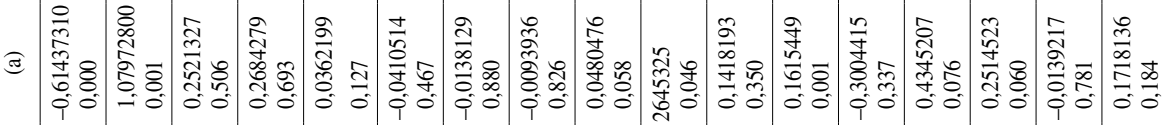


Becker, G. (1968), "Crime and punishment: an economic approach", Journal of Political Economy, vol. 76, Chicago, University of Chicago Press.

Benavente, J.M. y E. Melo (2006), "Determinantes socioeconómicos de la criminalidad en Chile durante los noventa", Documento de trabajo, $\mathrm{N}^{\circ}$ 223, Departamento de Economía, Universidad de Chile.

Block, M.K. y J.M. Heineke (1975), "A labor theoretic analysis of the criminal choice", American Economic Review, vol. 65, №3, Nashville, Tennessee, American Economic Association.

Buonanno, P. (2003a), "Crime, education and peer pressure", Working Paper, $\mathrm{N}^{\mathrm{o}}$ 64, Milán, Università degli Studi di Milano.

(2003b), "Identifying the effect of education on crime. Evidence from the Italian Regions", Working Paper, $\mathrm{N}^{\circ}$ 65, Milán, Università degli Studi di Milano.

(2003c), "The socioeconomic determinants of crime. A review of the literature", Working Paper, $\mathrm{N}^{\circ}$ 63, Milán, Università degli Studi di Milano.

Buonanno, P. y L. Leonida (2005), "Criminal activity and education evidence from Italian Regions", Working Paper, No 3, Bergamo, Università degli Studi di Bergamo.

Chambouleyron, A. y M. Willinton (1998), Crimen y castigo en Argentina: un enfoque empírico, Anales de la XXXII Reunión anual de la Asociación Argentina de Economía Política, Buenos Aires, Asociación Argentina de Economía Política.

Defensoría Penal Pública (2007), Informe estadístico anual 2006, Santiago de Chile.

Dryden Witte, A. (1980), "Estimating the economic model of crime with individual data", Quarterly Journal of Economics, vol. 94, $\mathrm{N}^{\mathrm{o}} 1$, Cambridge, Massachusetts, The MIT Press.

Ehrlich, I. (1973), "Participation in illegitimate activities: a theoretical and empirical investigation", Journal of Political Economy, vol. 81, $\mathrm{N}^{\circ} 3$, Chicago, University of Chicago Press.

Elias, D. y J. Okseniuk (2002), Racionamiento de crédito y regulación de capitales mínimos. Un análisis de corte transversal, Buenos Aires, Asociación Argentina de Economía Política.

Fajnzylber, P., D. Lederman y N. Loayza (2002), "Inequity and violent crime", Journal of Law and Economics, vol. 45, No 1, Chicago, University of Chicago Press.

Freeman, R. (1996), "Why do so many young American men commit crimes and what might we do about it?", Journal of Economic Perspectives, vol. 10, $\mathrm{N}^{\circ} 1$, Nashville, Tennessee, American Economic Association.
(1991), "Crime and the employment of disadvantaged youths", NBER Working paper, $\mathrm{N}^{\mathrm{o}} 3875$, Cambridge, Massachusetts, National Bureau of Economic Research.

Fundación Paz Ciudadana (2008), Anuario de estadísticas criminales 2008, Santiago de Chile.

Glaeser, E. y B. Sacerdote (1999), "Why is there more crime in cities?", Journal of Political Economy, vol. 107, № 6, Chicago, University of Chicago Press.

Imrohoroglu, A., A. Merlo y P. Rupert (2001), "What accounts for the decline in crime?", International Economic Review, vol. 45 $N^{\circ}$ 3, Filadelfia, University of Pennsylvania.

Lochner, I. (1999), "Education, work and crime: theory and evidence", RCER Working Paper, $\mathrm{N}^{\mathrm{o}} 465$, Rochester, Center for Economic Research, University of Rochester.

Lochner, I. y E. Moretti (2001), "The effect of education on crime evidence from inmates, arrest and self-reports", Working Paper, $\mathrm{N}^{\circ} 8605$, Cambridge, Massachusetts, National Bureau Economics Research.

Londoño, J., A. Gaviria y R. Guerrero (2000), Asalto al desarrollo: violencia y crimen en las Américas, Washington, D.C., Banco Interamericano de Desarrollo.

Nawata, K. y N. Nagase (1996), "Estimation of sample selection bias models", Econometric Reviews, vol. 15, No 4, Londres, Taylor \& Francis.

Núñez, J. y otros (2003), "Determinantes socioeconómicos y demográficos del crimen en Chile", Estudios de economía, vol. 30, No 1, Santiago de Chile, Departamento de Economía, Universidad de Chile.

Rivera, J., J. Núñez y X. Villavicencio (2004), “Crimen y disuasión: evidencia desde un modelo de ecuaciones simultáneas para las regiones de Chile", El trimestre económico, vol. 71, № 284 , México, D.F., Fondo de Cultura Económica.

Sah, R. (1991), "Social osmosis and pattern of crime", Journal of Political Economy, vol. 99, $\mathrm{N}^{\circ}$ 6, Chicago, The University of Chicago Press.

William, J. y R. Sickles (2002), "An analysis of the crime as work model: Evidence from the 1958 Philadelphia birth cohort study", Journal of Human Resources, vol. 37, № 3, Madison, University of Wisconsin Press.

Wolpin, K. (1980), "A time series-cross section analysis of international variations in crime and punishment", The Review of Economics and Statistics, vol. 62, $\mathrm{N}^{\circ}$ 3, Cambridge, Massachusetts, The MIT Press.

Wooldridge, J. (2001), Econometric Analysis of Cross Section and Panel Data, Cambridge, Massachusetts, The MIт Press. 